\title{
Spatio-temporal modelling of tick life-stage count data with spatially varying coefficients
}

\author{
Thabo Lephoto, ${ }^{1}$ Henry Mwambi, ${ }^{1}$ Oliver Bodhlyera, ${ }^{1}$ Holly Gaff ${ }^{2}$ \\ ${ }^{1}$ School of Mathematics, Statistics and Computer Science, University of KwaZulu-Natal, KwaZulu-Natal \\ Province, South Africa; ${ }^{2}$ Department of Biological Sciences, Old Dominion University, Norfolk, VA, USA
}

\begin{abstract}
There is a vast amount of geo-referenced data in many fields of study including ecological studies. Geo-referencing is usually by point referencing; that is, latitudes and longitudes or by areal referencing, which includes districts, counties, states, provinces and other administrative units. The availability of large geo-referenced datasets for modelling has necessitated the development and application of spatial statistical methods. However, spatial varying coefficients models exploring the abundance of tick counts remain limited. In this study we used data that was collected and prepared by researchers in the Department of Biological Sciences from the Old Dominion University, Virginia, USA. We modelled tick life-stage counts and abundance variability from 12 sampling locations, with 5 different habitats (numbered 1-5), three habitat types; namely: woods, edges and grass; collected monthly from May 2009 through December 2018. Spatio-temporal Poisson and spatio-temporal negative binomial (NB) count data models were fitted to the data and compared using the deviance informa-
\end{abstract}

Correspondence: Thabo Lephoto, University of KwaZulu-Natal, KwaZulu-Natal Province, Private Bag X01, Pietermaritzburg 3201, South Africa.

Tel.: +27.33.260.5785/+27.74.704.8528.

E-mail: LephotoT@ukzn.ac.za

Key words: Bayesian modelling; tick-counts; spatial and spatio-temporal effects.

Funding: this research was funded by NIH grant 1R01AI136035 as part of the joint NIH-NSF-USDA Ecology and Evolution of Infectious Diseases program. The funding body had no role in the design of the study and collection, analysis, and interpretation of data and in writing the manuscript.

Received for publication: 6 April 2021.

Revision received: 20 May 2021.

Accepted for publication: 20 May 2021.

(C) Copyright: the Author(s), 2021

Licensee PAGEPress, Italy

Geospatial Health 2021; 16:1004

doi:10.4081/gh.2021.1004

This article is distributed under the terms of the Creative Commons Attribution Noncommercial License (CC BY-NC 4.0) which permits any noncommercial use, distribution, and reproduction in any medium, provided the original author(s) and source are credited. tion criteria (DIC). The NB model outperformed the Poisson models with all its DIC values being smaller than those of the Poisson model. Results showed that the covariates varied spatially across counties. There was a decreasing time (in years) effect over the study period. However, even though the time effect was decreasing over the study period, space-time interaction effects were seen to be increasing over time in York County.

\section{Introduction}

Infectious agents that are transmitted by tick bites cause tickborne diseases, such as Rocky Mountain spotted fever, Lyme disease, Ehrlichiosis, tularaemia, babesiosis, Colorado tick fever, and relapsing fever (Tälleklint and Jaenson, 1998; Bowman et al., 2004; Bratton and Corey, 2005). Ticks are a highly specialized group of obligate, bloodsucking and non-permanent ectoparasitic arthropods that feed on mammals, birds and reptiles in most regions of the Earth. They are characterized by having relatively large body sizes among the acari; ingesting enormous quantities of vertebrate blood, digested tissues, or lymph; laying 200 to 23,000 eggs; and having moulting and reproduction regulated by blood ingestion (Anderson, 2002).

In Europe, ticks have spread and become established in areas that were previously not considered to be favourable for them. The reason is not only directly due to climate change, but also due to continuous changes in vegetation, landscape features and human social habits which are leading to new areas of contact between ticks, their pathogens and the interface between animals and humans (Salman, 2012). Tälleklint and Jaenson (1998) attribute these changes to climate change, which has led to the Ixodes ricinus species being detected in northern Sweden where they have now colonized relatively high altitude ranges in the mountains. In African countries, Asia, the Near East and parts of Europe, serious outbreaks of Crimean-Congo haemorrhagic fever have been reported. This fever is said to mainly be the result of tick-bites by Hyalomma marginatum, which is the most common tick species in Mediterranean-type environments and in the African steppes, but neither found in America nor Australia with absence of reported cases there to date (Salman, 2012).

Amblyomma americanum (lone star tick) is a major humanbiting tick in eastern, southern and mid-western U.S. (Goddard and Varela-Stokes, 2009). As in much of the world, tick-borne diseases are an emerging public health threat in this part of the U.S. (Sayler et al., 2017). According to Stromdahl and Hickling (2012), people who live and usually visit state parks and national forests in North America are at a higher risk of exposure to tick bites and their associated pathogens than those whose activities keep them outside of these areas. In this part of the U.S., there are only five 
tick species, i.e. A. americanum L., A. maculatum Koch, Dermacentor variabilis Say, Ixodes scapularis Say, and Rhipicephalus sanguineus, that commonly bite humans (Stromdahl and Hickling, 2012; Nathavitharana and Mitty, 2015). According to Childs and Paddock (2003), A. americanum is the primary vector for Ehrlichia chafleensis and E. ewingii, and it is associated with southern tick-associated rash illness and a number of other diseases. Despite its prominence as a nuisance biter and vector of human pathogens in the U.S., there is a lack of efforts to define the species' geographical range. This deprive us of information not only about identification of human populations at risk for A. americanum and its associated pathogens, but also in determining the landscape, host assemblage and environmental conditions that are favourable for the establishment and rapid reproduction of this tick species. It has emerged as one of the most important tick vectors in the U.S. transmitting pathogens to both humans and domestics animals (Childs and Paddock, 2003; Mixson et al., 2006; Goddard and Varela-Stokes, 2009; Fritzen et al., 2011). Hendricks et al. (2017) state that domestic dogs and cats are potentially effective sentinel populations for monitoring occurrence and spread of Lyme disease.

The focus of this study was to develop statistical models to model tick count data that include areal referencing. We wished to know whether the lone star tick life stage counts vary over time, across, and between, counties in south-eastern Virginia in the U.S. The lone star tick abundance is largely influenced by the availability of suitable animal hosts for the life stages and by the availability of habitats with physiographic features that offer protection for hosts and guard against desiccation of the tick (Childs and Paddock, 2003). Spatial and spatiotemporal statistics can reveal important environmental and temporal characteristics, for example, by incorporating time, space, and space time covariate interactions into the model. The main objective of this research was to use spatiotemporal analysis to explore environmental and temporal relationships with tick life-stage count data using data collected from 2009 through 2018. The knowledge and information unpacked are as essential in tick-related disease surveillance, as it is for effective planning and decision-making. This study could also be of interest with respect to the use of the INLA package in the R statistical software as compared to the Markov Chain Monte Carlo (MCMC) in the WinBUGS statistical software package.

\section{Materials and methods}

\section{Data source and study area}

We used data collected and prepared by researchers in the Department of Biological Sciences from Old Dominion University, Virginia. U.S. Ticks were collected using standard flagging techniques (CDC, 2020) along established transects and identified species and life-stage according to (Sonenshine, 1979). Larvae-, nymph- and adult-stage count data were recorded for each different location, habitat and habitat type throughout the four seasons of the year. Eight counties and independent cities were sampled in this state, namely; City of Chesapeake, City of Hampton, Isle of Wight County, City of Norfolk, Northampton County, City of Portsmouth, City of Virginia Beach and York County (Figure 1). For brevity, all will be referenced as counties. Data collection was done at least once a month on varying days of the week and at 12 different sites in southeast Virginia from May 2009 through
December 2018 (H. Gaff unpublished data). The data were collected at random from multiple areas referred to as habitats, and each area was designated by a unique number ranging from 1 to 5 for different habitats. The habitat type was used to designate the kind of area (woods, edges or grass) where the data were collected. The number of the week (from 1 to 53) was also recorded during data collection. Week 1 is the first week of the year, and while fewer ticks were observed in winter, the adult stage of I. scapularis is active in winter. To help align the information from year to year, we recorded data collected during the last week of December as week 53, which was also the first week of January of the following year. We also kept track of the month and year for the data collection. The ten-year study period was grouped into two-year successive periods such that 2009 and 2010 were grouped together, 2011 and 2012, 2013 and 2014, 2015 and 2016 and 2017 and 2018. This predictor variable was used to capture residual spatial effects on the abundance of tick counts. The paired time segmentation was to help find out if there were times when counts were significantly high or low compared with the rest of the data. The above-mentioned variables were used as predictors of tick life-stage count outcome data to find relationships using count regression models.

\section{Model descriptions}

Various statistical models have been developed to model count data. In this study, we applied the Poisson and the negative binomial distribution models. The classical Poisson model, which assumes that mean and variance of the count responses are equal, is often of limited use when the empirical data sets exhibit overdispersion or have more zeros than expected. This can be addressed by introducing a dispersion parameter in this model or by extension to models that can account for excessive zeros in the data (Zeileis et al., 2008). As mean and variance are identical in the standard Poisson model, this means that the dispersion parameter is fixed to 1. In the presence of over-dispersion, the Poisson model underestimates the variance and render all model-based tests more conservative. Violation of the equal mean and variance assumption indicates correlation in the data, which may affect both standard errors of the parameter estimates and the model. In this study, the tick count data showed greater proportion of zeros than that of positive counts, which is an indication of zero-inflation and over-dispersion.

The negative binomial (NB) distribution is commonly used to model over-dispersed count data. A dispersion parameter in the NB model caters for over-dispersion allowing the variance to be greater than the mean while also accommodating the unobserved heterogeneity in the data. In addition to over-dispersion, it is common that many empirical count data sets exhibit more zero observations than would be expected by the classical Poisson model. A model capable of capturing both properties is the zero-inflated Poisson (ZIP), which assumes that zero counts occur with some probability, while a Possion $(\lambda)$ random variable is observed with probability $1-p$. The ZIP distribution model approaches the classical Poisson distribution when $p \rightarrow 0$. It is worth noting that zero observations arise from both the zero-component distribution and the classical Poisson distribution. The zero-component distribution accounts for the inflated zeros that are observed in addition to zeros that are expected to be observed under the classical Poisson distribution. A more detailed account of the development of zeroinflated models can be found in (Lambert, 1992).

Let $y_{i j k m}$ be a tick count for life-stage $m(m=1$ : larvae; $m=2$ : nymphs; and $m=3$ : adults) in habitat-type $k$, habitat $j$ and location $i$ modelled by the multi-hierarchical Poisson model. Under the Poisson model, we assume that the dependent variable $y_{i j k m}$ is 
Poisson-distributed, i.e.

$y_{i j k m} \mid \mu_{i j k m} \sim \operatorname{Poisson}\left(\mu_{i j k m}\right)$

where $\mu_{i j k m}$ is the mean tick count in the respective life-stage, habitat type, habitat and location.

Let $X_{i j k m}=\left(\alpha_{m} ; x_{i j k m 1}, x_{i j k m 2}, \ldots, x_{i j k m p}\right)$ ' be a vector of $p$ continuous predictors with the first component accounting for the constant and $W_{i j k m}=\left(w_{i j k m 1}, w_{i j k m 2}, \ldots, w_{i j k m r}\right)$ ' be a vector of $r$ categorical predictors. The link function $h(\cdot)$ relates the mean $\mu_{i j k m}$ to the predictors as follows:

$h\left(\mu_{i j k m}\right)=X^{\prime} \beta_{m}+W^{\prime} \gamma_{m}$

where $W=W_{i j k m}$ and $X=X_{i j k m}$; while $h(\cdot)$ is the log link function, $\beta_{m}$ an m-dimensional vector of regression coefficients for continuous predictors; and $\gamma_{m}$ a vector of the categorical predictors.

In order to cater for non-linear effects of the continuous covariates, the spatial autocorrelation, and temporal effects in the data, we incorporated the random walk model of order 2 (RW2); the convolution models; the linear trend over years; and the space-time interactions into the model such that (Eq. 1) becomes:

$h\left(\mu_{i j k m}\right)=\beta_{0 m}+\sum_{i=1}^{p} f_{i}\left(S_{i}, x_{i j k m}\right)+f_{\text {month }}(m)+f_{\text {spat }}\left(S_{i m}\right)+f_{\text {year }}(t)+f_{i t}$ $\left(S_{i}, t\right)$

where the function $f_{i}\left(S_{i}, x_{i j k m}\right)$ represents the space-covariate interaction; $f_{\text {month }}(m)$ a non-linear twice differentiable smooth function for the continuous month covariate effect; and the functions $f_{\text {spat }}$ $\left(S_{i m}\right), f_{\text {year }}(t)$ and $f_{i t}\left(S_{i}, t\right)$ functions for space, time (years) and space-time interaction, respectively. The convolution model assumes that the spatial effect can be decomposed into two; namely, the spatially structured and spatially unstructured components. This means that $f_{\text {spat }}\left(S_{\text {im }}\right)=f_{\text {str }}\left(S_{\text {im }}\right)+f_{\text {unstr }}\left(S_{\text {im }}\right)$ where $m=1,2$ or 3 (Manda and Leyland, 2007; Ngesa et al., 2014; Okango et al., 2015).

The spatially structured random effects account for the unobserved covariates, which vary spatially across counties, while the spatially unstructured random effects account for the unobserved covariates that are inherent within counties or correlations within counties, for example, the climate and common cultural practices among other things. The spatially structured random effects are the spatial autocorrelations and they are technically defined as the correlation computed among the values of a single geo-referenced variable that is attributable to the geographic proximity of the objects, to which the values are attached (Cliff and Ord, 1981). Moreover, $f_{\text {year }}(t)$ represents random time effects which can be modeled as a first-order random walk (RW1) or a first-order autoregressive process (AR1), while $f_{i t}\left(S_{i}, t\right)$ is a space-time interaction.

In the presence of over-dispersion, the Poisson model is replaced by the negative binomial model, where the variance depends on the mean as $\mu(1+\mu \Psi)$, and where $\Psi$ is an over-dispersion parameter, which measures the extent at which variance deviates from the mean. We assumed that a tick count variable $y_{i j k m}$ follows a negative binomial distribution, such that

$y_{i j k m} \mid \mu_{i j k m} \sim \mathrm{NB}(\mu, \Psi)$

The mean function $E\left(y_{i j k m}\right)=\mu_{i j k m}$ relates to the predictors in the same way as that of the Poisson distribution model in Eq. 1. In this study, a full Bayesian estimation approach was used, where parameters were assigned prior distributions.

\section{Model diagnostics}

The models were compared using the deviance information criterion (DIC), which is obtained by adding the posterior mean of the deviance that measures the goodness of fit to the number of effective parameters as: $D I C=\bar{D}(\theta)+p_{D}$ where $\bar{D}$ is the posterior mean deviance and $p_{D}$ is the effective number of parameters in the model, which penalizes the fit for complexity of the model. Spiegelhalter et al. (2002) state that $p_{D}$ values less than zero indicate substantial conflict between the prior and the data or that the posterior mean is a poor estimator. The best model is said to be the one with the smallest DIC value. Low values of $\bar{D}$ suggest a better fit, while small values of $p_{D}$ suggest model parsimony as discussed in Spiegelhalter et al. (2002).

\section{Fitted models}

Four spatio-temporal models were fitted in the $\mathrm{R}$ statistical software, version 3.5.1 using the 'INLA' package to predict the effects of the ecological covariates on the distribution of larvae, nymph and adult tick counts in the eight previously mentioned counties of Virginia, U.S. Preparation, organization and merging of the data with the map was done using the QGIS software, version 3.6.3-Noosa (QGIS, 2009). The first approach modelled the spacecovariate effects $f\left(S_{i}, x_{i j k m}\right)$, non-linear effects of the month covariate, $f_{\text {month }}(m)$, the temporal time effects $f_{\text {year }}(t)$ and the space-time effects $f_{i t}\left(S_{i}, t\right)$. This model (M1) does not consider the spatially structured and spatially unstructured random effects and the three life-stage counts were modelled independently as follows:

M1: $\log \left(\mu_{i j k m}\right)=\beta_{0 m}+\sum_{i=1}^{p} f\left(S_{i}, x_{i j k m}\right)+f_{\text {month }}(m)+f_{\text {year }}(t)+f_{i t}\left(S_{i}, t\right)$

where $m$ equals 1 for larvae; 2 for nymphs; and 3 for adults with respect to counts. The second model (M2) is the same as M1 but with spatially unstructured effects to cater for the unobserved covariates that are inherent within the counties. The spatially unstructured effects were specified by the identically and independent distributed (iid) with the normal distribution.

M2: $\log \left(\mu_{i j k m}\right)=\log \left(\mu_{i j k m}\right)=\beta_{0 m}+\sum_{i=1}^{p} f\left(S_{i}, x_{i j k m}\right)+f($ month $)+f_{\text {unstr }}$ $\left(S_{i}\right)+f_{\text {year }}(t)+f_{i t}\left(S_{i}, t\right)$

The third model (M3) is the same as M1 but with spatially structured effects which cater for any unobserved covariates which vary spatially across counties, specified by the conditional autoregressive model (CAR).

M3: $\log \left(\mu_{i j k m}\right)=\log \left(\mu_{i j k m}\right)=\beta_{0 m}+\sum_{i=1}^{p} f\left(S_{i}, x_{i j k m}\right)+f($ month $)+f_{s t r}$ $\left(S_{i}\right)+f_{\text {year }}(t)+f_{i t}\left(S_{i}, t\right)$

The fourth model (M4) is the same as M1 with a convolution of spatially unstructured and spatially structured effects, which are specified by the iid normal distribution and CAR model respectively.

M4: $\log \left(\mu_{i j k m}\right)=\log \left(\mu_{i j k m}\right)=\beta_{0 m}+\sum_{i=1}^{p} f\left(S_{i}, x_{i j k m}\right)+f($ month $)+f_{\text {unstr }}$ $\left(S_{i}\right)+f_{\text {str }}\left(S_{i}\right)+f_{\text {year }}(t)+f_{i t}\left(S_{i}, t\right)$

We used the Poisson and the negative binomial count data distributions and compared them using the DIC. We then interpreted the results from the best performing models based on the DIC. 


\section{Assessment}

Before presenting the study results, we first performed an exploratory data analysis and also compared all the models using the DIC. We compared spatio-temporal Poisson models with their corresponding spatio-temporal negative binomial models.

\section{Exploratory data analysis}

Table 1 shows environmental and time predictor variables used in this study. Table 2 shows the number of positive and zero counts in the data. It is obvious that the data have more zero counts than positive counts for all tick life stages.

Our records show that the larvae were more abundant in the environment than the nymph and adult stage ticks at all times. As seen in Figure 2, the blue bars for larvae are on average, approximately 7 and 14 times taller when compared with the orange and grey bars for the nymph and adults, respectively, during the study period from 2009 through 2018.

\section{Model comparisons}

Table 3 shows the DICs for four spatio-temporal Poisson and NB models, where the model with the smallest DIC is the one with the best fit. As seen, all the spatio-temporal NB models have lower DICs compared to the corresponding spatio-temporal Poisson models. M1 showed the best fit for the tick larvae count data compared to the all the other models, which also suggests that unobserved covariates vary significantly over time. Similarly, M4 showed the best fit for the nymph and adult tick count data. The differences in the DIC values suggest that the spatio-temporal NB model would be the best model compared to the spatio-temporal Poisson model. This outcome also suggests that unobserved covariates vary spatially across counties and over time.

\section{Results}

\section{Space-covariate effects}

Spatio-temporal NB models out-performed the spatio-tempo- ral Poisson models in our case. We show the choropleth maps (Figures 2-4) of all the models with the smallest DICs for the fitted larvae, nymph and adult tick count data, respectively. The choropleth maps show the space-covariate interaction effects for the selected counties in Virginia. A yellow shade was used if the effects are greater and black or dark shade if the effects were lower.

\section{Larvae}

The effect of location on the log mean tick counts was highest in Hampton County followed by York and Chesapeake counties (Figure 3). The effect was lower in Norfolk, Portsmouth and Isle of Wight counties. Virginia Beach County showed the lowest effect of the location variable. The effect of habitat was almost the same across all the counties except for Isle of Wight County. The effect of change in weeks was evident in York and Norfolk counties. The effect of habitat type on the log of mean larvae tick counts was high in Chesapeake County. The effect of change in season was very low in Isle of Wight County compared to other counties.

Table 1. Predictor variables and their types.

\begin{tabular}{lc} 
Predictor & Type \\
Habitat & Environmental \\
Habitat type & Environmental \\
\hline Location & Environmental \\
Year & Time \\
\hline Month & Time \\
Season & Time \\
\hline
\end{tabular}

Table 2. Tick counts in the data, 2009-2018.

\begin{tabular}{lcc} 
Variable & Positive counts & Nil counts \\
Larvae & 726 & 3767 \\
Nymphs & 1770 & 2723 \\
\hline Adults & 1365 & 3128 \\
\hline
\end{tabular}

Table 3. Deviance information criteria values for spatio-temporal Poisson and negative binomial models.

\begin{tabular}{|c|c|c|c|c|c|c|}
\hline \multirow[t]{3}{*}{ Response } & \multirow{3}{*}{ Total counts } & \multirow{3}{*}{ Model } & \multicolumn{4}{|c|}{ Spatio-temporal models } \\
\hline & & & & & & \\
\hline & & & DIC & $p_{D}$ & DIC & $p_{D}$ \\
\hline Larvae & 145020 & & 281147.18 & 71.79 & 12563.79 & 13.31 \\
\hline Nymphs & 20637 & 1 & 29982.53 & 79.52 & - & - \\
\hline Adult & 10509 & & 13990.35 & 62.55 & - & - \\
\hline Larvae & 145020 & & 277483.97 & 73.81 & 12591.82 & 9.64 \\
\hline Nymphs & 20637 & 2 & 30176.78 & 67.67 & 14203.41 & 49.58 \\
\hline Adult & 10509 & & 14308.39 & 62.55 & 10101.87 & 47.52 \\
\hline Larvae & 145020 & & 295800.95 & 71.16 & 12600.72 & 10.82 \\
\hline Nymphs & 20637 & 3 & 34093.74 & 65.24 & 14518.67 & 45.97 \\
\hline Adult & 10509 & & 14317.57 & 62.60 & 10101.87 & 47.52 \\
\hline Larvae & 145020 & & 277468.27 & 73.88 & 12591.73 & 9.64 \\
\hline Nymphs & 20637 & 4 & 30176.53 & 67.64 & 14202.29 & 49.36 \\
\hline Adult & 10509 & & 14308.23 & 62.55 & 10100.97 & 47.13 \\
\hline
\end{tabular}

DIC, deviance information criteria; NB, negative binomial. 


\section{Nymphs}

The effect of location on the log mean nymph counts was high in Hampton County and very low in Norfolk County compared to the others (Figure 4). The effect of habitat was very low in Isle of Wight County, and low in Northampton County followed by Chesapeake, Hampton and Portsmouth counties. However, there was high effect of habitat in Norfolk County. The effect of change in week was very low in York County and Northampton County but high in Portsmouth County. The effect of habitat type on the log nymphal counts was evident in Chesapeake County but low in Isle of Wight County. The effect of change in seasons was very low in Isle of Wight County, while a higher effect can be observed in York, Hampton and Norfolk counties (Figure 4).

\section{Adults}

The effect of location on the log mean adult counts was high in York County, while it was generally low in other counties. The effect of habitat was high in Norfolk and Hampton counties, but it was a very low in Isle of Wight County. The effect of change in week was also evident in the latter. A very low effect in this respect can be observed in York County (Figure 5). The effect of habitat type was very high in Chesapeake County and very low in York County compared with other counties. The effect of change in season was very high in Norfolk County and very low in Isle of Wight County. Other counties had low effects as compared to the effect in the latter.

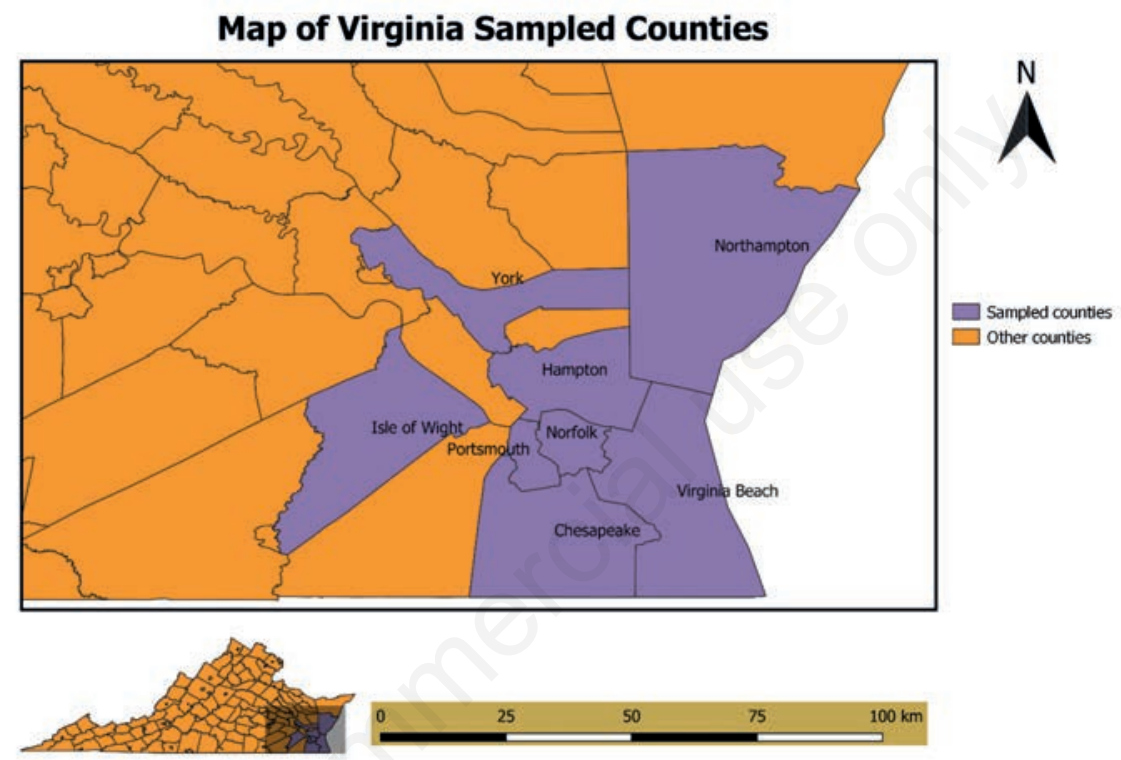

Figure 1. Map of Virginia with names of counties where ticks were sampled.

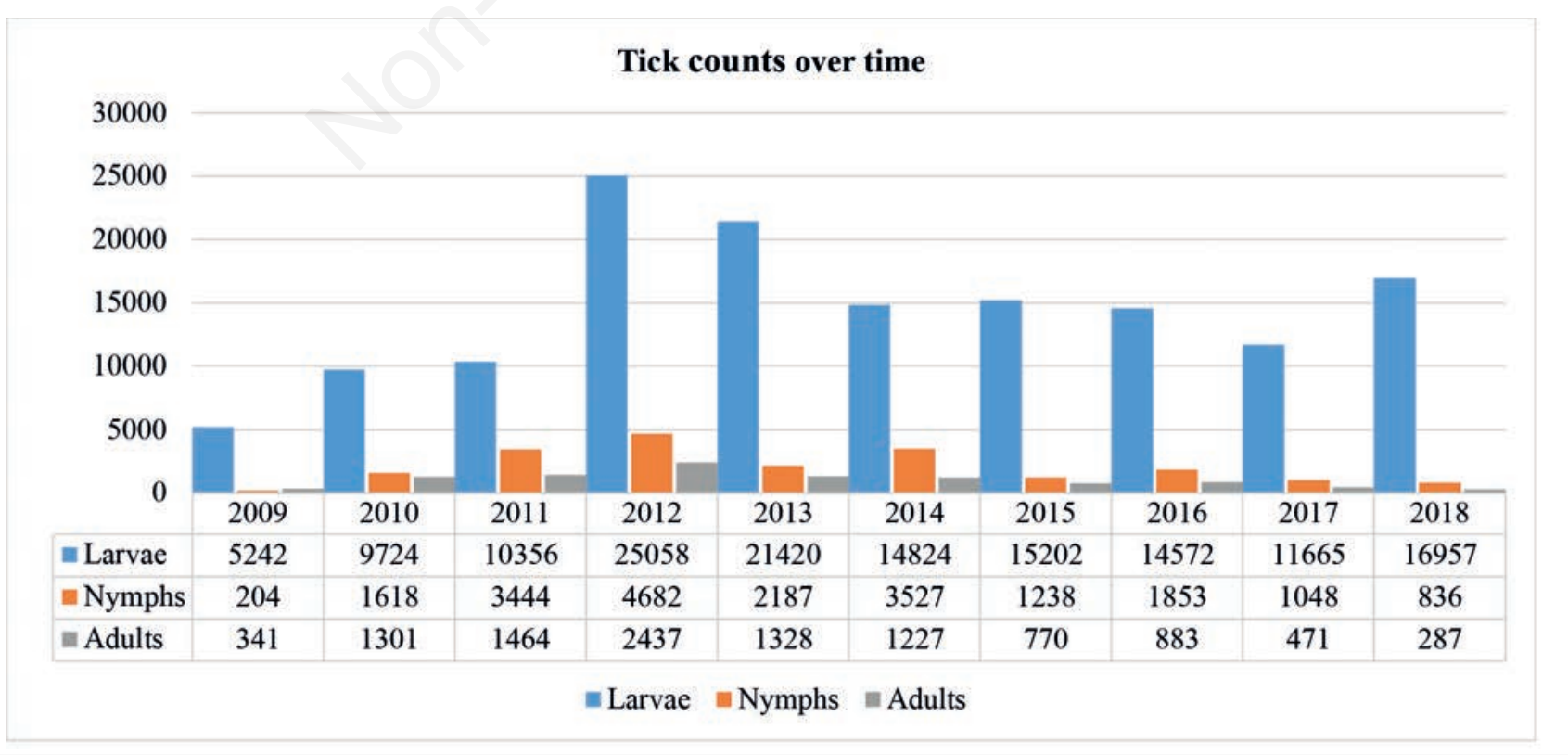

Figure 2. Tick counts of larvae, nymph and adult species in Virginia, USA 2009-2018. 
The non-linear effects of the month

Figure 6 shows non-linear associations between time (month) and larvae, nymph and adult tick counts. These figures give the posterior mean (black line in the figure) of the smooth function and their corresponding 95\% credible intervals (red and blue lines). From the figures it is evident that there is a non-linear relationship between month and tick counts.

We could confirm the general changes along the seasons in the study area. Thus we report the probability of observing larvae increases between January and April, fluctuates between April and June and then starts to increase even further between June and August. After those chances of observing larvae decrease until December. The chances of observing nymphs increase slightly between January and February, the number of larvae increases abruptly between February and June, then decreases again between June and August followed by a steady decrease between August and September. From there, it decreases sharply between September and December. The chances of observing adults increases steadily between January and February, then increases
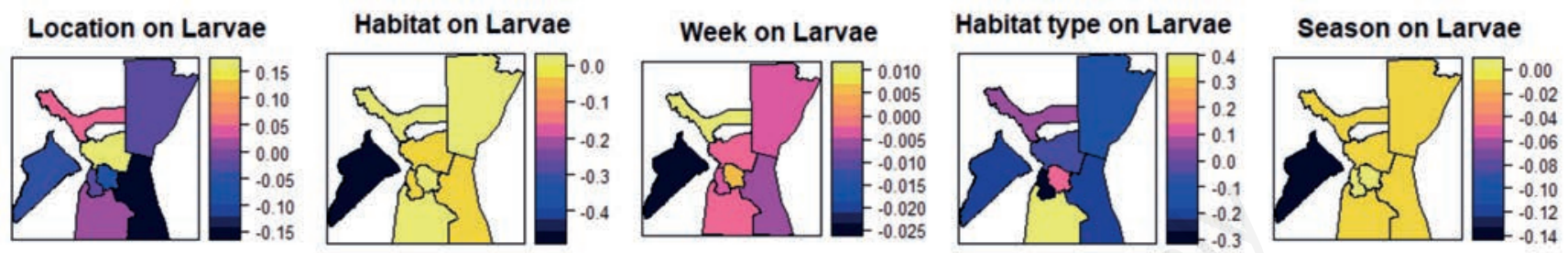

Figure 3. Choropleth maps showing the space-covariate effects on larvae.

\section{Location on Nymphs}

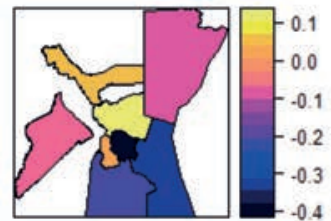

\section{Habitat on Nymphs}

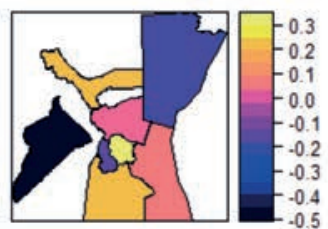

Week on Nymphs

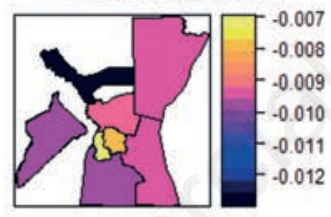

Habitat type on Nymphs

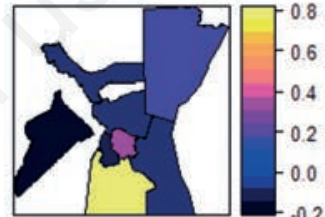

Season on Nymphs

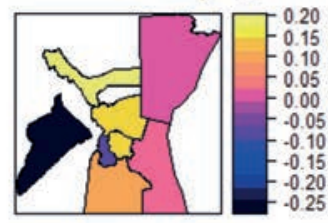

Figure 4. Choropleth maps showing the space-covariate effects on nymphs.

Location on Adults

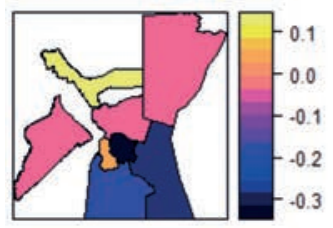

Habitat on Adults

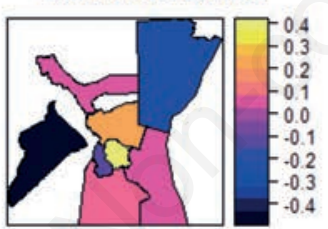

Week on Adults

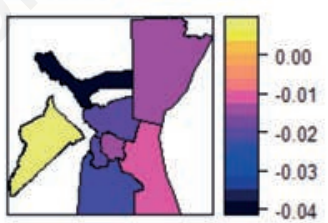

Habitat type on Adults

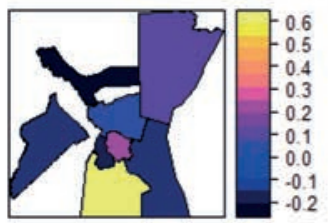

Season on Adults

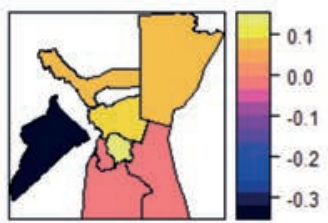

Figure 5. Choropleth maps showing the space-covariate effects on adults.
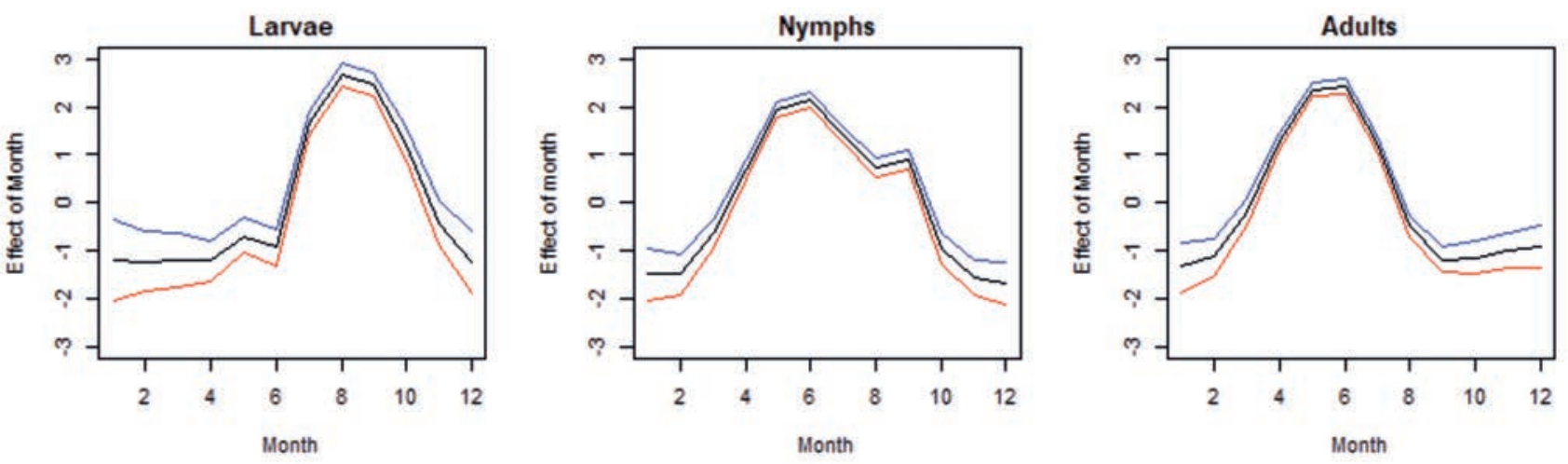

Figure 6. Non-linear effects of month variable on larvae, nymphs and adults 2009-2018. 
abruptly from February to May, steadily between May and June and decreases sharply between June to September. The probability then starts to increase steadily from September to December.

\section{Space-time effects}

\section{Larvae}

Figure 7 shows the mapped estimated residual spatial effects on the abundance of larvae counts between 2009 and 2018. The residual spatial effects that represent unobserved spatial factors either not measured in the surveys or abducting the effects of cultural patterns are evident. High effects were observed in Portsmouth, Chesapeake and Hampton counties in 2009/2010 while the other counties showed low residual spatial effects. Three counties showed decreased residual spatial effects in the period 2011/2012 and these effects were evident in Norfolk and Northampton counties. In the period 2013/2014 the effects were evident in Hampton, Portsmouth, Chesapeake and York counties. The effects were high in
Chesapeake, Norfolk and York counties 2015/2016, while they were very low in Portsmouth County. During 2017/2018 all counties, except for Isle of Wight and Portsmouth counties, showed high effects on the abundance of larvae.

\section{Nymphs}

The effects on the nymph abundance decreased over the whole study period, particularly in York County (Figure 8).

\section{Adults}

Residual spatial effects on the distribution of abundance of adult ticks increased between 2009 and 2016 in York County, while all other counties showed a decrease of spatial residual effects throughout the study period 2019 to 2018 . In York, a decrease in residual spatial effects was only observed after 2015/2016 study period. Higher residual spatial effects were evident in Norfolk County during the periods 2009/2010, 2011/2012 and 2013/2014, respectively (Figure 9).

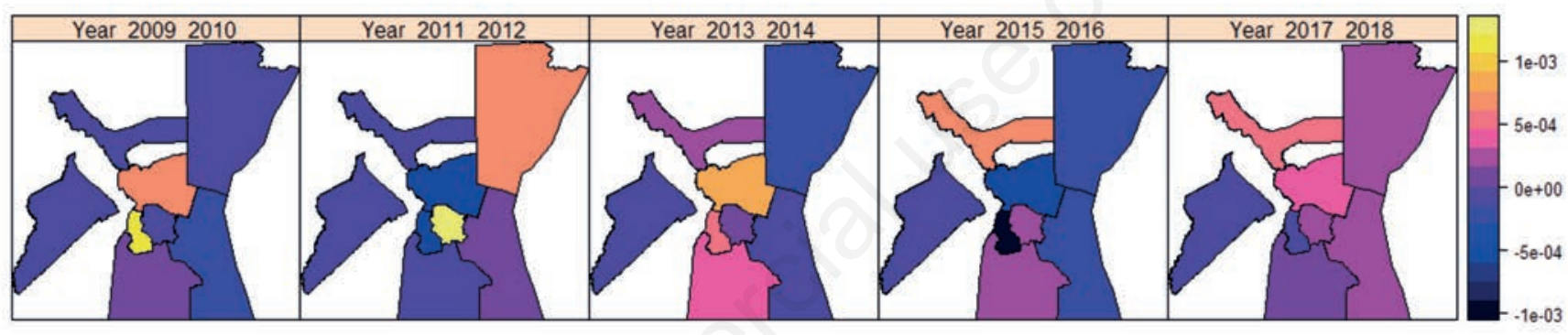

Figure 7. Choropleth maps showing residual spatial effect of larvae tick abundance 2009-2018.

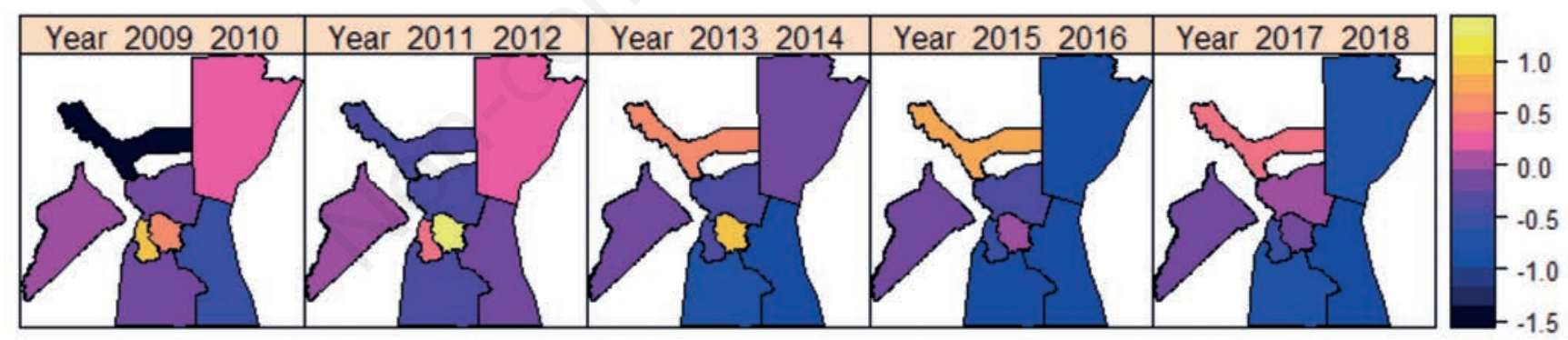

Figure 8. Choropleth maps showing residual spatial effect of nymph tick abundance 2009-2018.

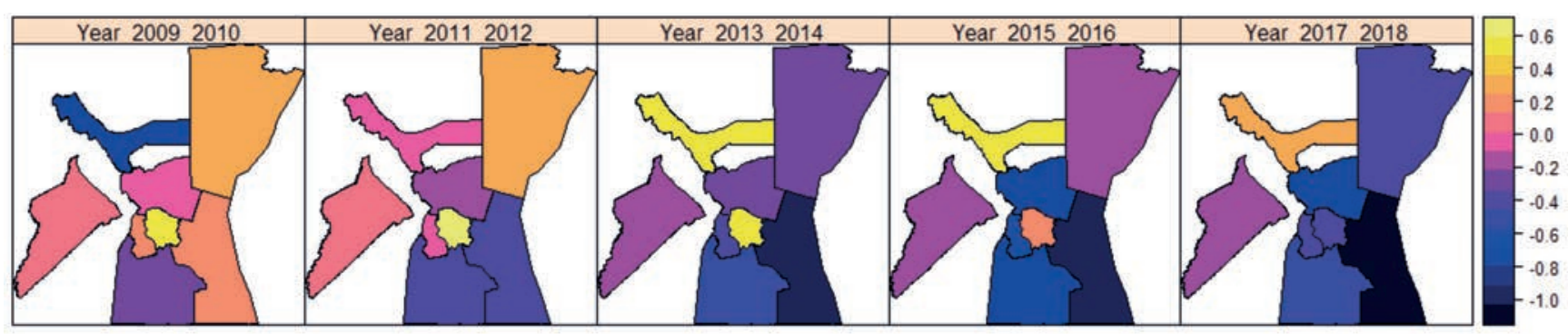

Figure 9. Choropleth maps showing residual spatial effect of adult tick abundance 2009-2018. 


\section{Temporal effects}

For larvae, there was a steady decreased in the mean count between periods 1 and 5 , then a steady increase from period 4 . The graph indicates that larvae abundance declined between 2009 and 2016, after which there was a steady increase in the abundance larvae ticks. The graph indicates that nymph abundance increased between periods 2009 and 2012 then declined between 2012 and 2016, after which there was a steady increase in the abundance of nymph ticks. For adults, the abundance was constant from 2009 through 2012, after which a gradual decline was observed from 2012 until 2018 (Figure 10).

\section{Discussion}

This study applied the Poisson and NB count data models to tick count data with the aim to explore the influence of environmental and temporal predictors on the distribution of tick counts in Virginia, U.S. We relaxed the assumption that the relationship between the predictors and the response variables in a regression model are constant across the study region and over time. This assumption is unrealistic for spatial processes as factors such as sampling variation and different relationships across regions, for example, attitudes, preferences, culture and others, contribute to different responses to the same stimuli as one moves across regions and over time. A frequent approach to spatial modelling dates back to the work by Besag et al. (1991) which was extended by Bernardinelli et al. (1995) to include a linear term for space-time interaction. Many studies have relaxed this assumption, e.g., Assunçao et al. (1999) introduced a Bayesian space-varying parameter model to examine micro-region factor productivity and the degree of factor substitution in the Brazilian agriculture; Gamerman et al. (2003) developed a flexible modelling approach for space-varying regression models; and Okango et al. (2015) modelled the HIV and HSV-2 viruses using spatially varying coefficient models. The Bayesian spatio-temporal process model was used to allow covariates to vary spatially and over time. We specified the CAR prior for the structured random effects; autoregressive of order one (AR1) prior for the temporal random effects; and normal iid priors for the unstructured random effects. Non-linear effects of the month variable on tick counts were also evident. As a result, an assumption of linear relationship would have led to misleading results and consequently to wrong interpretations.

The exploratory data analysis showed that more larvae counts were observed compared with nymphs and adult tick counts. We found that the effects of covariates on tick counts varied spatially across counties and over time. Spatio-temporal models were powerful, in a sense that they were capable of revealing county specific effects of each covariate, county space-time effects and the effects of change in time on the distribution of life-stages of lone star ticks. We were able to show that tick abundance has been increasing over the study time in Virginia, which confirms the previous results by Lantos et al. (2015) that observed the significant expansion of Lyme disease distribution in Virginia between 2000 and 2014, particularly southward into the Virginia mountain ranges.

It is clear that change in temperature affect tick numbers, such that they decrease in winter (Linske et al., 2020). We confirmed this unsurprising fact and noted also that larvae count remained low up to May. Non-linear effects of the month showed that nymphs and adults were observed in spring and summer, meaning that the distribution of larvae would be expected to increase later that summer and thus adults the following spring. This happens in locations early summer thus determining the distribution of larvae and adults in late summer and spring the following year, respectively (Stein et al., 2008).

Our study also revealed that the effects of habitat type were high in Chesapeake County. This could be because of the abundant white-tailed deer population found in forests, farms, parks and backyards throughout the Chesapeake Bay Watershed. The lone star tick is said to be very aggressive and specific when looking for hosts (Goddard and Varela-Stokes, 2009), but they are unspecific during each life-stage, as this species is found on humans, domesticated animals, ground-dwelling birds as well as on small and large wild animals (Sonenshine and Stout, 1971; Kollars, 1993). The white-tailed deer feeds on fruits and vegetation that are available to them each season, which makes it simple for ticks to attach and feed from these animals. During warm seasons (summer and spring), these animals feed on green plants, during fall they feed on nuts, acorns and crops and in winter they feed on woody vegetation, such as bark, twigs and buds of hardwood and pine trees, where indeed ticks are found (Willis et al., 2012).

\section{Limitations}

The failure to account for excess zeros in the discrete distribu-
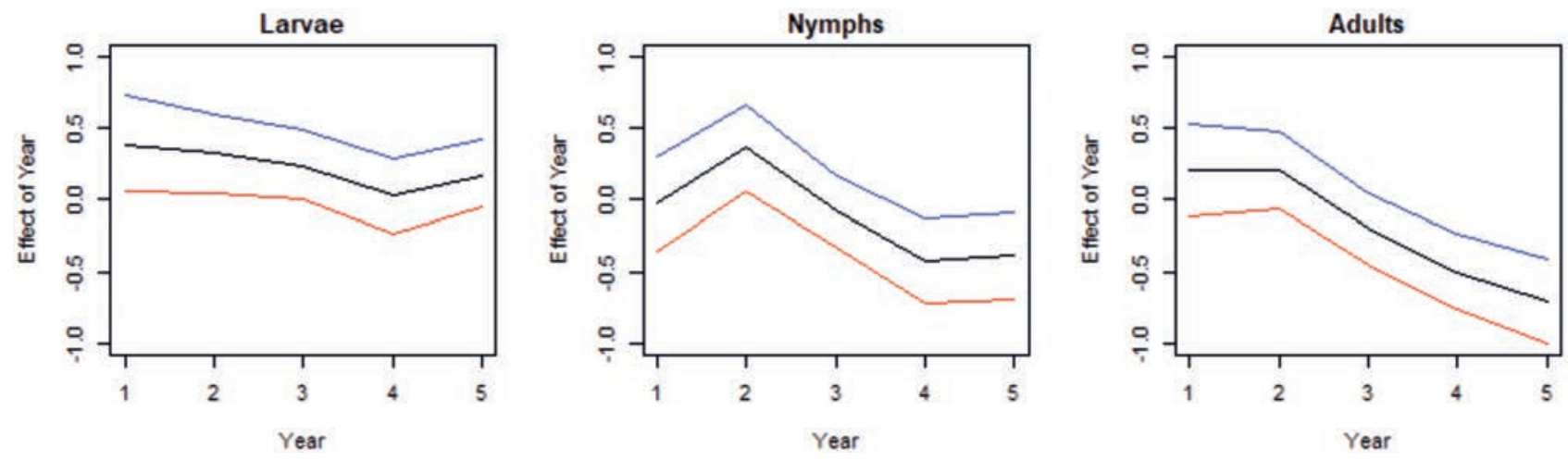

Figure 10. The temporal year random effect for the cumulative best fitting model. Black line = the estimated posterior mean; blue and red lines $=$ the upper and lower $95 \%$ credible interval. 
tions was a problem. The study focused only on the univariate independent models rather than joint multivariate modelling of counts. In the future, zero-inflated models should be developed and applied to the data. Covariate-time-space interaction effects could also be incorporated. This interaction is able to show the effects of predictors of tick life-stage counts in space and over time. Due to limited data, the study only looked at three environmental and two time predictor variables.

\section{Conclusions}

Tick counts are influenced by environmental factors and seasonal changes. There is no dominant weekly influence or observable change in the number of ticks due to the changing weeks. We conclude that tick numbers depend on the type of habitat where they are closer to their hosts and the time when their hosts are more likely to be targeted. Grassy and wooded places are the most liked by ticks as hosts feed and live in such places. Larvae counts are to be expected during summer between June and August, while nymphs are found in abundance between February and May, while adult counts appear mainly between February and May.

\section{References}

Anderson JF, 2002. The natural history of ticks. Med Clin North Am 86:205-18.

Assunçao J, Gamerman D, Assunçao R, 1999. Regional differences in factor productivities of Brazilian agriculture: a space varying parameter approach. In Proceedings of the XV Latin American Meeting of the Econometric Society.

Bernardinelli L, Clayton D, Montomoli C, 1995. Bayesian estimates of disease maps: how important are priors? Stat Med 14:2411-31.

Besag J, York J, Mollié A, 1991. Bayesian image restoration, with two applications in spatial statistics. Ann Inst Stat Math 43:1-20.

Bowman AS, Nuttall PA, Chappell L, 2004. Ticks: biology, disease and control. Parasitol 129:S1-S1.

Bratton RL, Corey GR, 2005. Tick-borne disease. Am Fam Physician 71:2323-30.

CDC, 2020. Guide to the surveillance of metastriate ticks (Acari: Ixodidae) and their pathogens in the U.S. Division of VectorBorne Diseases, CDC, Atlanta \& Ft. Collings.

Childs JE, Paddock CD, 2003. The ascendancy of Amblyomma americanum as a vector of pathogens affecting humans in the United States. Annu Rev Entomol 48:307-37.

Cliff AD, Ord JK, 1981. Spatial processes: models \& applications. Taylor \& Francis, Boca Raton, FL, USA.

Fritzen CM, Huang J, Westby K, Freye JD, Dunlap B, Yabsley MJ, Schardein M, Dunn JR, Jones TF, Moncayo AC, 2011. Infection prevalences of common tick-borne pathogens in adult lone star ticks (Amblyomma americanum) and American dog ticks (Dermacentor variabilis) in Kentucky. Am J Trop Med Hyg 85:718-23.

Gamerman D, Moreira AR, Rue H, 2003. Space-varying regression models: specifications and simulation. Comput Stat Data Anal 42:513-33.

Goddard J, Varela-Stokes AS, 2009. Role of the lone star tick, Amblyomma americanum (L.), in human and animal diseases. Vet Parasitol 160:1-12.

Hendricks B, Mark-Carew M, Conley J, 2017. Evaluating the util- ity of companion animal tick surveillance practices for monitoring spread and occurrence of human Lyme disease in West Virginia, 2014-2016. Geospat Health 12:582.

Kollars TM, 1993. Ticks (Acari: Ixodidae) infesting medium-sized wild mammals in southwestern Tennessee. J Med Entomol 30:896-900.

Lambert D, 1992. Zero-inflated Poisson regression, with an application to defects in manufacturing. Technometrics 34:1-14.

Lantos PM, Nigrovic LE, Auwaerter PG, Fowler Jr VG, Ruffin F, Brinkerhoff RJ, Reber J, Williams C, Broyhill J, Pan WK, 2015. Geographic expansion of Lyme disease in the southeastern United States, 2000-2014. In Open forum infectious diseases, pp. ofv143. Oxford University Press.

Linske MA, Williams SC, Stafford KC, Lubelczyk CB, Henderson EF, Welch M, Teel PD, 2020. Determining effects of winter weather conditions on adult Amblyomma americanum (Acari: Ixodidae) survival in Connecticut and Maine, USA. Insects 11:13.

Manda SO, Leyland A, 2007. An empirical comparison of maximum likelihood and Bayesian estimation methods for multivariate disease mapping: theory and methods. S Afr Stat J 41:1-21.

Mixson TR, Campbell SR, Gill JS, Ginsberg HS, Reichard MV, Schulze TL, Dasch GA, 2006. Prevalence of Ehrlichia, Borrelia, and Rickettsial agents in Amblyomma americanum (Acari: Ixodidae) collected from nine states. J Med Entomol 43:1261-8.

Nathavitharana RR, Mitty JA, 2015. Diseases from North America: focus on tick-borne infections. Clin Med (Northfield Il) $15: 74$.

Ngesa O, Mwambi H, Achia T, 2014. Bayesian spatial semi-parametric modeling of HIV variation in Kenya. PLoS One 9:e103299.

Okango E, Mwambi H, Ngesa O, Achia T, 2015. Semi-parametric spatial joint modeling of HIV and HSV-2 among women in Kenya. PLoS One 10:e135212.

QGIS DT, 2009. QGIS Geographic Information System, Open Source Geospatial Foundation.

Salman MD, 2012. Ticks and tick-borne diseases: geographical distribution and control strategies in the Euro-Asia region; CABI. Available from: https:/www.cabi.org/bookshop/ book/9781845938536/

Sayler K, Rowland J, Boyce C, Weeks E, 2017. Borrelia burgdorferi DNA absent, multiple Rickettsia spp. DNA present in ticks collected from a teaching forest in North Central Florida. Ticks Tick Borne Dis 8:53-9.

Sonenshine, D. E. (1979) Insects of Virginia: No. 13: Ticks of virginia (Acari, Metastigmata). Research Division Bulletin 139, Department of Entomology, Virginia Polytechnic Institute and State University, Blacksburg, VA, USA.

Sonenshine DE, Stout IJ, 1971. Ticks infesting medium-sized wild mammals in two forest localities in Virginia (Acarina: Ixodidae). J Med Entomol 8:217-27.

Spiegelhalter DJ, Best NG, Carlin BP, Van Der Linde A, 2002. Bayesian measures of model complexity and fit. J Roy Stat Soc Ser B (Stat Method) 64:583-639.

Stein KJ, Waterman M, Waldon JL, 2008. The effects of vegetation density and habitat disturbance on the spatial distribution of ixodid ticks (Acari: Ixodidae). Geospat Health 2:241-52.

Stromdahl E, Hickling G, 2012. Beyond Lyme: aetiology of tickborne human diseases with emphasis on the South-Eastern United States. Zoonoses Public Health 59:48-64.

Tälleklint L, Jaenson TG, 1998. Increasing geographical distribu- 
tion and density of Ixodes ricinus (Acari: Ixodidae) in central and northern Sweden. J Med Entomol 35:521-6.

Willis D, Carter R, Murdock C, Blair B, 2012. Relationship between habitat type, fire frequency, and Amblyomma ameri- canum populations in east-central Alabama. J Vector Ecol 37:373-81.

Zeileis A, Kleiber C, Jackman S, 2008. Regression models for count data in R. J Stat Softw 27:1-25. 